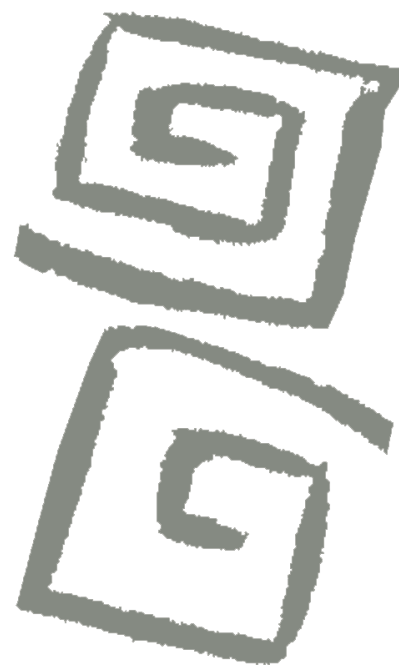

\title{
Orgullo loco y metáforas para una disidencia: un análisis lingüístico y simbólico
}

\author{
Mad pride and metaphors for dissidence: a linguistic \\ and symbolic analysis
}

Martín Correa-Urquiza Vidal', Asun Pié Balaguer ${ }^{2}$, Marta Coll-Florit', Eulàlia Hernández i Encuentra ${ }^{4}$, Salvador Climent Roca ${ }^{5}$

'Doctor en Antropología Médica y Salud Internacional. Profesor Asociado, Universitat Rovira i Virgili, Tarragona, España. $\bowtie$ i iD

Doctora en Pedagogía. Profesora Agregada, Universitat Oberta de Catalunya, Barcelona, España. $\bowtie$ iD

${ }^{3}$ Doctora en Sociedad de la Información y el Conocimiento, especialidad en Lingüística Aplicada. Profesora Agregada, Universitat Oberta de Catalunya, Barcelona, España. $\triangle$ (iD)

${ }^{4}$ Doctora en Psicología. Profesora Agregada. Universitat Oberta de Catalunya. Barcelona, España. $\Delta$ (iD)

${ }^{5}$ Autor de correspondencia. Doctor en Ciencia Cognitiva Lenguaje. Profesor Agregado. Universitat Oberta de Catalunya. Barcelona, España. $\triangle$ iD
RESUMEN El presente artículo expone un análisis lingüístico e interpretativo sobre el uso de la metáfora conceptual en el campo de la salud mental, tomando como campo de observación el uso de Twitter en la primera edición del Día del Orgullo Loco en España, celebrada el 20 de mayo de 2018. El objetivo es dar cuenta de los posicionamientos expresados por los activistas en primera persona. Los resultados muestran un cuestionamiento a las lógicas coercitivas producidas por la atención psiquiátrica, una problematización del modelo hegemónico en su conjunto, una denuncia a la opresión que implica el estigma, problemas de comunicación y demandas de mayor diálogo con los profesionales del campo de la salud mental. Este análisis nos ha permitido comprender el modo lingüístico de re-semantizar el campo de la salud mental, así como dar cuenta de las tensiones existentes entre las percepciones subjetivas de las personas diagnosticadas y las producciones del modelo médico hegemónico.

PALABRAS CLAVES Salud Mental; Metáfora; Redes Sociales; Estigma Social; Disidencias; España.

ABSTRACT This paper presents a linguistic and interpretative analysis of the use of conceptual metaphors in the field of mental health, taking as a field of observation the use of Twitter in the first edition of Mad Pride Day in Spain, held on May 20, 2018. The objective is to give a first-person account of the attitudes expressed by activists. The results show a questioning of the coercive logics produced by psychiatric care, a problematization of the hegemonic model as a whole, a criticism of the oppression implied by stigma, communication problems with professionals in the field of mental health, as well as demands for greater dialogue with them. This analysis has allowed us to understand linguistic modes of resignifying the field of mental health, and also to account for the tensions between subjective perceptions of the people diagnosed and the productions of the hegemonic medical model.

KEY WORDS Mental Health; Metaphor; Social Networking; Social Stigma; Dissent; Spain. 


\section{INTRODUCCIÓN}

Históricamente, el ámbito del sufrimiento psíquico y/o la salud mental ha sido un campo de análisis e intervención en manos de los saberes expertos que han ido operando sobre la experiencia de la aflicción, transformándola en una problemática objetivada, sujeta a la investigación y actuación de los profesionales del ámbito sanitario, psicológico y/o social. Intervenciones que se han ejercido habitualmente desde un marco de referencia unidimensional, sostenido en las lógicas hegemónicas del paradigma biomédico. Dicha situación no ha estado exenta de contradicciones y fue generando resistencias, disidencias y movimientos contrahegemónicos de diversa naturaleza ${ }^{(1,2,3,4)}$. En este sentido, una de las corrientes con más resonancias en los últimos años es la que resulta del llamado movimiento en primera persona, cuyas articulaciones se fundamentan en la reivindicación de un lugar central para los sujetos de la aflicción en la elaboración de los discursos y prácticas que construyen, tanto los sentidos sociales y clínicos otorgados al sufrimiento psíquico como la terapéutica y los itinerarios de la recuperación. Dicho movimiento no es uniforme sino diverso y se materializa a partir de entidades, asociaciones o colectivos, "en ocasiones con posicionamientos muy diferentes en lo que respecta a la negociación con los saberes y las lógicas hegemónicas del cuidado, pero unidos en la reivindicación de sus derechos ciudadanos como uno de los elementos que desnaturalizan y cuestionan las metodologías manicomiales persistentes en ciertos territorios y vínculos institucionales"(4). Es un conjunto heterogéneo, que si bien en ocasiones reproduce los desencuentros y conflictos internos de las instituciones y prácticas a las que interpela, ha encontrado en la celebración anual del Día del Orgullo Loco un espacio de unidad, enunciación y reivindicación nunca antes visto en el ámbito de la salud mental.

El Día del Orgullo Loco es un momento festivo pero, fundamentalmente, un episodio de cuestionamiento a la preeminencia del relato biomédico y a sus impactos en la reproducción de las lógicas del estigma; un grito común a modo de cirugía epistemológica sobre lo dado. No se trata tanto de una negación del sufrimiento o de las complejas situaciones sintomatológicas, sino de un cuestionamiento a los contextos y a los marcos de referencia a través de los cuales son leídas, nombradas, tratadas las aflicciones y sus síntomas. En el Día del Orgullo Loco se producen pancartas y carteles, se corean consignas desde megáfonos y se utilizan, sobre todo, los recursos de las redes sociales -Twitter fundamentalmente- como altavoces y multiplicadores de mensajes, como instrumentos desde donde anunciar y enunciar lo que se reivindica y cuestiona. Allí, ese día, lo que se coloca en disputa es el campo de significaciones a partir del cual se piensa el sufrimiento psíquico y, por lo tanto, la jurisdicción sobre los itinerarios posibles de los sujetos que se derivan a partir de ello.

El trabajo aquí presentado se enmarca en MOMENT: Metáforas del trastorno mental grave $^{(5)}$, proyecto de investigación de carácter interdisciplinar, formado por lingüistas e investigadores en salud mental de diversos ámbitos (antropología médica, psicología y educación social), cuyo objetivo fundamental es analizar los discursos sobre salud mental generados en Internet por los dos grandes colectivos implicados: personas diagnosticadas con un trastorno mental grave y profesionales de la salud mental. En este marco, el presente artículo busca analizar y problematizar lo dicho, expresado y exclamado por el primero de estos colectivos, tomando como campo de observación el uso de Twitter durante la primera edición del Día del Orgullo Loco celebrado en el estado español, el día 20 de mayo de 2018. A través del análisis lingüístico, busca observar las maneras a partir de las cuales las metáforas utilizadas durante el evento manifiestan un determinado posicionamiento, una necesidad expresada que interpela al modelo hegemónico, una denuncia a la opresión que implica el estigma, un cuestionamiento a las lógicas coercitivas que en ocasiones se reproducen en la atención psiquiátrica y una exigencia de participar en la construcción dialógica de los itinerarios y 
prácticas para la producción del mejor estar psíquico de las poblaciones.

\section{La celebración}

En el estado español existe una ceremonia promovida desde las instituciones de la red de salud mental y las federaciones de familiares, que tiene lugar cada 10 de octubre. Es un festejo enmarcado en el Día Mundial de la Salud Mental celebrado en más de 100 países y apoyado por la Federación Mundial de la Salud Mental. Por el contrario, la celebración del Día del Orgullo Loco es un movimiento independiente que intenta dar un lugar central a las reivindicaciones y acciones de las mismas personas con sufrimiento psíquico diagnosticado, nucleando a gran parte del movimiento en primera persona. Dicha conmemoración que surgió aquí en el año 2018, se suma y se inscribe a su vez en una lógica estatal y global, ligada a movimientos que desde la última década del siglo XX comenzaron a gestarse en países como Brasil, Canadá, Reino Unido, Francia y Chile, entre otros.

El 20 de mayo de 2018, la celebración se realizó entre actividades, disertaciones desde tarimas, acciones reivindicativas y una serie de manifestaciones que recorrían espacios centrales de algunas de las principales ciudades del estado. El lema fue "El orgullo lo cura" y su objetivo, según reza en la misma página web de la jornada, se centró en visibilizar y romper prejuicios alrededor de la salud mental. El Día del Orgullo Loco no fue sino una gran plataforma de expresión colectiva, que planteaba la necesidad anunciada de dejar de ser objetos de la intervención clínica, para ser sujetos de acción y diálogo ante sus propios procesos de recuperación y empoderamiento, actores en sus itinerarios de diagnosis, tratamiento, pronóstico y vida. Ante todo esto, Twitter fue altavoz, y no solo contribuyó a darle visibilidad al evento potenciando y ampliando el alcance de los mensajes, sino que operó como agente productor de encuentros, adhesiones y multiplicación.

\section{METODOLOGÍA Y CORPUS DE ESTUDIO}

En lingüística cognitiva se entiende por metáfora conceptual| ${ }^{(6)}$ el mecanismo mental con expresión lingüística mediante el cual se comprenden y expresan conceptos abstractos en términos de otros más concretos $\mathrm{y}$, a menudo, más cercanos a la experiencia. De esta manera, determinadas propiedades semánticas de los conceptos concretos se transfieren a los conceptos abstractos facilitando así su comprensión y la eficacia comunicativa. Este proceso suele ser inconsciente y suele pasar inadvertido para el hablante y para el oyente. Por ejemplo, en (1) se conceptualiza la vida con una enfermedad en términos de una gue$\mathrm{rra}^{(7)}$. Técnicamente, el mecanismo conceptual se suele anotar con fórmulas como $\mathrm{La}$ vida es una guerra, y se asume que está expresado lingüísticamente en la frase de ejemplo (y en otras muchas, ya que se trata de una metáfora muy productiva), tal como revela el uso de las palabras usadas metafóricamente "luchador", "enfrentarse" y "arma" (en este y sucesivos ejemplos, las palabras usadas metafóricamente se presentan subrayadas). El concepto explicado (en este caso, "la vida") se denomina dominio destino y el concepto con el que figuradamente se compara ("la guerra") se denomina dominio de origen.

(1)

Son grandes luchadores, se enfrentan con su enfermedad con las armas que la propia enfermedad les deja.

Para detectar en el corpus de estudio el lenguaje metafórico y formularlo para su análisis, se ha utilizado el método desarrollado por Coll-Florit y Climent ${ }^{(8)}$, el cual se basa en la identificación de palabras usadas metafóricamente y su extrapolación a metáforas conceptuales mediante el uso de diccionarios de referencia y repertorios de metáforas compilados a partir de estudios previos y bibliografía de referencia. 
El corpus se constituyó en torno al primer Día del Orgullo Loco, celebrado en España el 20 de mayo de 2018. Para ello se recogieron todos los tweets escritos en español durante el año 2018, que incluyeran al menos una mención de los usuarios y hashtags del conjunto listado en (2), más los que respondieran a uno de estos. El conjunto (2) consiste en los nombres de usuario de los organizadores del evento, más los hashtags por ellos propuestos para caracterizar los tweets relativos al evento. El corpus resultante consta de 3.174 tweets y 87.926 palabras.

(2)

@orgullo_loco, @orgullboig, $@$ OrgulloLocoMad, \#orgulloloco, \#EIOrgulloLoCura, \#20MYoVo, \#orgullboig, \#orgullfoll, \#20demaigboig, \#critsilenciat

Algunos estudios previos han analizado la metáfora en el ámbito de la salud mental, centrándose en diferentes tipos de corpus como grabaciones de sesiones de terapia ${ }^{(9)}$, entrevistas a personas afectadas ${ }^{(10)}$, programas de radio $^{(11)}$ o documentales sobre salud mental ${ }^{(7,12)}$. Asimismo, si bien Twitter también ha sido usado para el análisis de discursos vinculados a la salud mental como espacio de empoderamiento a partir de la construcción conjunta de discursos para la sensibilización y lucha contra el estigma ${ }^{(13,14)}$, hasta el momento no se ha realizado ningún estudio de estas características centrado en el análisis de metáforas conceptuales. Esta es, pues, una de las principales innovaciones del presente estudio.

El presente estudio se enmarca en el proyecto MOMENT: Metáforas del trastorno mental grave. Análisis del discurso de personas afectadas y profesionales de la salud mental, que recibió la aprobación del Comité de Ética de la Universitat Oberta de Catalunya el 14 de julio de 2018.

\section{ANÁLISIS DEL CORPUS}

En este apartado se ofrecen los resultados del análisis lingüístico e interpretativo del corpus que se centran, de acuerdo con el objetivo del trabajo, en las metáforas conceptuales. El conjunto de tweets contiene muestras abundantes de otros fenómenos lingüísticos como neologismos formales (por ejemplo, "farmafia", "trastornariado") y neologismos semánticos $^{(15)}$ (por ejemplo, "loco", en los que el sentido peyorativo se desactiva y se activa un sentido reivindicativo) o metonimias ${ }^{(16)}$ (por ejemplo, voz por derecho a opinar); sin embargo, estos fenómenos no se analizarán aquí, para centrarnos en el fenómeno discursivo de la metáfora conceptual.

Sobre un total de 3.174 tweets $(87.926$ palabras) se han detectado 1.455 metáforas del ámbito de la salud mental, lo que arroja una ratio de 0,45 metáforas por tweet (aproximadamente, una metáfora cada dos tweets). Distinguiremos, en primer lugar, entre los antes mencionados dominios de origen y dominios destino. El dominio destino es indicativo de qué temas tratan las metáforas de los mensajes, es decir, aquellos aspectos de la problemática que resultan relevantes para el emisor, y el dominio de origen indica en qué términos se conceptualizan dichos temas.

Como puede verse en la Tabla 1, que recoge el número de expresiones metafóricas que incluyen un determinado dominio destino con más de 50 ocurrencias en el corpus, los ámbitos más conceptualizados son, con diferencia, la vida de la persona diagnosticada y las profesiones relacionadas con

Tabla 1. Metáforas conceptuales: principales dominios de destino.

\begin{tabular}{lc} 
Dominio destino & $\begin{array}{c}\text { Expresiones } \\
\text { metafóricas }\end{array}$ \\
Vida de la persona diagnosticada & 323 \\
Profesión & 196 \\
Comunicación & 116 \\
\hline Prejuicio social & 93 \\
\hline Sistema & 59 \\
\hline Trastorno & 58 \\
Persona diagnosticada & 56
\end{tabular}

Fuente: Elaboración propia a partir de datos obtenidos en el presente estudio. 
la salud mental. A continuación, se sitúan las conceptualizaciones relativas a aspectos comunicativos del afectado y al prejuicio social. Las metáforas sobre el trastorno, la persona diagnosticada como individuo y la superestructura social que los activistas denominan sistema tienen también una presencia importante.

Las metáforas de "la vida de la persona diagnosticada" conceptualizan el proceso de vivir con un diagnóstico de trastorno mental (como veremos más abajo: principalmente en términos de "guerra" o "viaje"); las metáforas de "la persona diagnosticada" responden a conceptualizaciones de la propia persona en tanto entidad; y las del "trastorno" corresponden a reificaciones de la afectación mental. Respecto al dominio destino "profesión", en la inmensa mayoría de casos (154), las expresiones se refieren explícitamente a la psiquiatría y, en otro importante volumen de casos (20), refieren a un profesional médico sin especificar, siendo presumible, por el contexto, que se trata de psiquiatras. Las referencias explícitas a otras profesiones (enfermeros, psicólogos, profesionales de la industria farmacéutica) son marginales.

Por lo que respecta a los dominios de origen, los presentaremos en relación al dominio destino que conceptualizan, es decir, formulados ya como metáforas. La Tabla 2 muestra las metáforas predominantes en el corpus (más de 40 expresiones metafóricas).

Tabla 2. Metáforas conceptuales predominantes.

\begin{tabular}{|c|c|}
\hline Metáfora & Número \\
\hline La vida de la persona diagnosticada es una guerra & 193 \\
\hline La profesión es poder* & 121 \\
\hline La vida de la persona diagnosticada es un viaje & 96 \\
\hline El prejuicio social es una marca & 74 \\
\hline Comunicación/incomunicación es mostrarse/esconderse & 72 \\
\hline Comunicación/incomunicación es proximidad/distancia & 44 \\
\hline
\end{tabular}

A continuación, se presentan los tipos mayoritarios de metáforas detectados en el corpus, seguidos de su interpretación.

\section{"La vida de la persona diagnosticada es una guerra"}

De manera abrumadoramente mayoritaria, la vida de la persona diagnosticada se conceptualiza en términos de una guerra o conflicto (193 expresiones metafóricas), o de un viaje (96 expresiones metafóricas); el resto de dominios de origen de la vida del diagnosticado son residuales (menos de 10 expresiones metafóricas). La metáfora de la vida de la persona con diagnóstico como "guerra" se realiza en muchas expresiones metafóricas en que no se lexicaliza un enemigo especificado (3a), por lo que se puede interpretar como relativa al esfuerzo y el conflicto inherentes al activismo. Sin embargo, cuando el enemigo se especifica, este suele ser el estigma (3b, 3c).

(3)

a. Luchando con \#OrgulloLoco por la
dignidad de TODAS. \#1diciembreyovoy

b. Pero quedó en la trinchera gente validísima y hoy, gracias en parte a ellos, gracias a lo que él empezó, ahora tenemosa@FlipasGAM, al @OrgulloLocoMad, a@Gacela1980... defendiendo unos derechos que se siguen negando y apedreando al estigma.

c. \#orgulloloco \#madpride La batalla contra un estigma que afecta al $20 \%$ de la población

Esta metáfora da cuenta de la tensión y, a menudo, la colisión entre la realidad percibida por las personas diagnosticadas, el sentido común y las prácticas clínicas de la red de salud mental. Para la fenomenología, lo real se crea en el consenso y el encuentro de subjetividades, en diálogo, creando una suerte de "sentido común" sobre lo real ${ }^{(17)}$. Este sentido común, que se adquiere a través de la educación, la religión y la cultura, está implicado 
en la construcción de la hegemonía de modo dialógico ${ }^{(18)}$ y supone que los valores y la visión del mundo de las clases dominantes (hegemónicas) se conviertan en el sentido común compartido para el resto de grupos (subalternos). Para Martínez-Hernáez ${ }^{(19)}$, la locura no se opone a la razón, sino al sentido común. Nos dice que la locura está relacionada con algo obsceno (fuera de escena) porque rompe con este sentido común, producto de las tensiones producidas por la hegemonía. Justamente lo que irrita y espanta de la locura es el colapso que produce en la convención social ${ }^{(17)}$. Pone en cuestión un arbitrio naturalizado que, de otro modo, difícilmente sería cuestionado.

En primer lugar, la colisión entre las experiencias en primera persona y este sentido común (convención social) señala la arbitrariedad de las normas sociales y la producción e impacto del estigma. El estigma es la consecuencia directa del imaginario social hegemónico actuando sobre la Ilamada enfermedad mental. Opera como una marca existencial en el conjunto de sus vidas: "Este estigma persigue al paciente prácticamente toda su vida, es difícil sacarse el mote de loca o de loco. Uno tiene miedo de contar que estuvo allí dentro (refiriéndose al psiquiátrico)"(20). Refiere, por lo tanto, a la iatrogenia o efecto nocebo del campo social.

El campo de las prácticas biomédicas, por su lado, desactiva la desnormalización producida por la propia locura, por la vía de la enfermedad o el organicismo extremo. El cuerpo emerge entonces como destino insoslayable siendo la propia existencia un acto político. Existir es resistir. La especificidad de las etiquetas nosológicas en el campo de la salud mental está relacionada con el hecho de que no solo no conllevan un tratamiento curativo (se fundan en la cronicidad), sino que además imposibilitan inventar tránsitos saludables en el afuera de la red de salud mental. El sujeto queda colonizado, enteramente cuestionado. En este sentido, la apropiación del lenguaje impulsa la generación de otros espacios, liminares, fronterizos que resisten al monolítico dentro o fuera. Entre el binomio enfermo mental o cuerdo se habilita un tercer espacio (loco), donde es posible habitar la palabra y, en consecuencia, abrir otro sentido común: el de la locura. Este territorio de frontera es campo de enfrentamientos, resistencias y luchas. La noción de locura es polisémica, se abre a otras posibilidades interpretativas; no queda ya clausurada en la lógica de lo patológico, sino que se expone a nuevas aperturas conceptuales que permiten otras libertades. El tercer espacio es aquel en el que se vuelve posible un cierto margen de elección y emancipación de lo dado, en el que la locura se propone como idea que puede ser en tanto generadora de colectivo, identidades y resistencias. La metáfora de la guerra nos explica el sentir de las personas con diagnóstico, así como las luchas cotidianas a las que se ven obligadas a enfrentarse pero, particularmente, nos indica el nivel de violencia simbólica y real que viven. Esta es la segunda colisión y refiere a la gestión personal de la violencia sufrida por el sistema de salud mental. Numerosos trabajos describen en primera persona este nivel de violencia vinculado a las propias lógicas manicomiales, tanto es así que en muchos casos hablan de tortura y de poder sobrevivir a la psiquiatría: "Al respecto, cabe señalar que la sobremedicación, las prácticas de internación involuntaria y tratamiento psiquiátrico forzoso, aplicación de electroshock, medidas de contención física, aislamiento social, abandono, sobreprotección, tortura y tratos abusivos, entre otras violaciones a los derechos humanos en el campo de la salud mental, son cotidianas..."(21).

Al mismo tiempo, puede deducirse de lo enunciado que, para las personas diagnosticadas, la vida es una guerra, pero no necesariamente en lo relativo a su realidad psíquica compleja o las circunstancias de sus aflicciones derivadas del trastorno, sino contra el despojo de derechos, contra la fosilización identitaria dentro de las lógicas circulares de la enfermedad, contra la deslegitimación social para pensar y producir curas y cuidados. Es una guerra y, al mismo tiempo, un intento de emancipación de lo hegemónico, un grito para dejar de ser solo objeto de intervenciones, sino sujeto de su propia experiencia y 
recuperación. La metáfora de la guerra se asienta y es consecuencia de una realidad que ya sostenía Michel Foucault, entre otros, cuando afirmaba en El poder psiquiátrico:

De tu sufrimiento y tu singularidad sabemos cosas suficientes (que ni sospechas) para reconocer que son una enfermedad; pero conocemos esa enfermedad lo bastante como para saber que no puedes ejercer sobre ella y con respecto a ella ningún derecho. Nuestra ciencia permite llamar enfermedad a tu locura, y por ello, nosotros, los médicos, estamos calificados para intervenir y diagnosticar en ti una locura que te impide ser un enfermo como los demás: serás por lo tanto un enfermo mental. ${ }^{(3)}$

\section{"La vida de la persona diagnosticada es un viaje"}

Por lo que respecta a la conceptualización de la vida de la persona diagnosticada como viaje, sus manifestaciones son muy diversas, con gran variedad de compañeros de viaje (4a), como un profesional o la familia, y obstáculos $(4 \mathrm{~b}, 4 \mathrm{c})$ como la estigmatización, que puede ser uno de ellos. Pero, en cualquier caso, se conceptualiza mejorar como avanzar (4d) y el activismo como el camino a seguir (4e).

(4)

a. Sobre la medicación (o desmedicalización) nunca daría consejos, solo puedo hablar de mi experiencia, a mi me acompañó una psiquiatra en todo el proceso.

b. Cada cual tiene sus procesos, lo sé. Pero ojalá que mucha gente que hoy está en el \#MeMad pueda tuitear pronto con \#MadPride. El camino pasa por el \#orguIloloco y la superación del autoestigma

c. ¿A quiénes le interesa que impere la normalidad? Es hora de cuestionarnos cómo nos oprime la normalidad y esta puede convertirse en un obstáculo para el desarrollo personal \#EIOrgulloLoCura
@orgullo_loco,@orgull_boig,@FlipasGAM, @FliparteGAM

d. Y dicho esto, me parece una pasada que haya gente que dé pasos, aunque sean pequeñitos, en hacer entender nuestro sufrimiento y educar a los sanitarios... Es un avance.

e. Trabajar en colectivo es jodido, pero es el camino que hemos elegido. Quienes no saben o no quieren currar en colectivo carecen de legitimidad para hablar de espacios comunes.

La vida del diagnosticado como viaje pone de manifiesto, en muchos casos, las alianzas activistas y sus estrategias en los procesos de recuperación. Estos procesos se conceptualizan en ocasiones como nuevas formas de subjetividad que requieren de determinadas condiciones sociales para su advenimiento. Estas condiciones se ubican en el afuera de la red hegemónica de salud mental, pero también, en ocasiones, en el afuera de los proyectos antimanicomiales orquestados por profesionales. Este viaje o tránsito se concibe dentro del activismo como un proceso de liberación. Las ideas de avanzar están relacionadas con la recuperación de la propia agencia y también con la transformación del sistema de salud mental:

\footnotetext{
El sistema por sí solo no va a generar algo distinto. Eso distinto hay que generarlo fuera del sistema. Afuera del sistema se puede liberar a las personas físicamente, moralmente, intelectualmente para que hagan con sus vidas lo que quieran, para que no estén secuestradas por miedos, por la ignorancia, por las farmacéuticas, por los intereses de los psiquiatras, de los psicólogos, y todas las capas medias de profesionales que administran a este sector. $^{(22)}$
}

En la metáfora "la vida es un viaje" se vislumbra también la idea de proceso, de vulnerabilidad y necesidad de atravesar instancias que requieren del cuidado mutuo, lo colectivo, el 
diálogo con los saberes expertos. Como nos diría Freire ${ }^{(23)}$, la liberación se produce en grupo o no se produce. Existe por tanto un trabajo colectivo que se inicia con el sujeto político de la locura y que conlleva necesariamente un componente dialógico y una trama de redes de sostén. El cuidado es dialéctico. Es un ida y vuelta entre las diferentes perspectivas posibles en torno a las circunstancias de la aflicción lo que contribuiría a la consecución de un bienestar.

\section{"La profesión es poder"}

La conceptualización de las profesiones relacionadas con la salud mental se concentra en presentarlas como un poder que se ejerce sobre la persona afectada (121 de las 196 expresiones metafóricas relativas a profesionales de la salud mental) y, muy especialmente, conceptualizando la psiquiatría como un poder represor (110 de estas 121).

El análisis de las correspondencias ontológicas o implicaciones de la metáfora revelan que la concepción del sistema psiquiátrico como un poder represor tiene distintos aspectos derivados: la persona diagnosticada es un ser subordinado (5a), distintas prácticas médico-hospitalarias se conciben como tortura (5b) y el propio ejercicio médico como violencia (5c).

a. Me colocaron en esa posición receptora, patologizada, sumisa/sometida. Ahora me nombro como loca, mujer psiquiatrizada, diagnosticada, con sufrimiento psíquico... No uso "enferma"

b. La primera vez casi no sobrevivo a la tortura psiquiátrica, pensé que lo que me ocurrió era una horrible excepción. Pero atar a las personas sin recibir la atención adecuada, sobremedicar y anularte como persona es lo que recibimos cuando pedimos ayuda. \#70añosvulnerados

c. Por eso, que estar a la vez con agresores y agredidos se me hace extraño e incoherente, pero allá vosotros con vuestras decisiones, claro (y allá la gente que elija compartir con vosotros proyectos y mañana llaméis otra vez a otro que defienda atarnos por lo de dar voz a todos)"

De manera relacionada, la industria farmacéutica se conceptualiza también en diversos casos como un ente represor o directamente como una organización criminal (6).

(6)

Medicamentos que matan y crimen organizado y Psicofármacos que matan y denegación organizada.

Esta metáfora está relacionada con la anterior "la vida de la persona diagnosticada es una guerra" $y$, particularmente, da cuenta del campo relacional en la red de salud mental. La imposición de los tratamientos no es algo excepcional, de modo que los mecanismos de persuasión, manipulación y coerción son múltiples y variados. El ejercicio de poder es vertical, centrado en el síntoma y el efecto del psicofármaco. No existe el diálogo, sino el monólogo de los saberes expertos, la circularidad interpretativa a partir de la cual toda enunciación por parte de la persona con diagnóstico es interpretada en términos de sintomatología de la enfermedad ${ }^{(24)}$. Las metáforas dominantes son la forma que toma la resistencia frente a la unidimensionalidad interpretativa de la fenomenología de la locura.

El poder no solo se da entre profesionales y usuarios sino también intradisciplinariamente. Ello está relacionado con la jerarquización de saberes. Saberes menos válidos que otros ocupan los escalafones inferiores y, entre ellos, el más denostado es el saber lego. Por ello la (re) apropiación del lenguaje resulta crucial para lograr una apertura semántica y con ella un lugar social para la diversidad. La estructura que sostiene y reproduce el ejercicio de poder vertical es el modelo biomédico hegemónico que, según Menéndez, se define, entre otras características, por estar centrado en una aproximación biologicista y a-historicista de los procesos de salud/enfermedad, y de articularse desde una jerarquización estructural 
entre saberes expertos y saberes legos ${ }^{(25)}$. Por ello, muchas voces insisten en que la transformación del sistema no puede lograrse desde dentro, sino que es necesario construir un afuera formado de otros espacios, otras palabras y otros saberes. Un ejercicio del poder más horizontal va inextricablemente unido a una recomposición dialógica entre saberes. Pero, además, el hecho de que exista una renuncia al poder o la generación de un espacio compartido de diálogo y toma de decisiones también va inevitablemente unido al cambio de la propia identidad profesional y en consecuencia a una renuncia. Para que los usuarios amplíen su campo de decisión, los profesionales deben renunciar a la unilateralidad. Ello significa que deben aceptar una mayor complejidad en la toma de decisiones, una descentralización del fármaco a favor de las narrativas subjetivas (otros modos de nombrar y contar) y en definitiva un cambio en la cultura asistencial. Es precisamente esto lo que se promueve el Día del Orgullo Loco.

\section{"El prejuicio es una marca"}

En cuanto al prejuicio social, es muy notorio el uso reiterado del término "estigma" (y derivados morfológicos como "autoestigma", "estigmatizar" o "desestigmatizar") (7a). La creciente convencionalización de este término en el ámbito de la salud mental (y no solo en este ámbito) no debe hacer olvidar su carácter metafórico, dado que su significado básico remite a una marca física indeleble, ignominiosa o infligida como signo de esclavitud. Otro conjunto más reducido de expresiones igualmente evoca, mediante el término "etiqueta" (7b), una marca que señala socialmente a la persona diagnosticada. En conjunto, estas expresiones metafóricas conforman una metáfora productiva que podemos denominar "el prejuicio social es una marca".

(7)

a. Si lees el comunicado y a perfiles como @Lo_co_munu@OrgulloLocoMadverás que la intención es de reivindicación, empoderamiento y desestigmatización.

b. Vamos a acabar con vuestras etiquetas, vamos a crear una nueva realidad social donde sentir y percibir diferente no sea motivo de tortura y exclusión porque el \#OrgulloLoco ha nacido para hacer HISTORIA y de nuestra locura brotará una sociedad más justa \#SaludMental \#Psicosis360 pic.twitter.com/gREoWJGMYy

En relación con los términos "estigma" y "etiqueta", debe hacerse notar que reaparecen en otras metáforas, lo que sin duda señala su relevancia para los activistas de Orgullo Loco. En primer lugar, como hemos apuntado, el "estigma" aparece como enemigo en la metáfora de guerra (3) y como obstáculo en la metáfora de viaje (4), lo que remarca aún más el destacado carácter negativo de la estigmatización para los activistas. "Etiqueta", por su parte, es un término que, aunque como acabamos de ver en algunos casos denota el prejuicio social, más habitualmente refiere al diagnóstico médico (8) lo que, juntamente con otras expresiones, motiva la metáfora "el diagnóstico es una marca".

(8)

Las locas también saldremos el 20M a la calle a exigir que no vulneren nuestros derechos solo por decidir etiquetarnos con i1, 2, 3? diagnósticos. A gritar que cuando la locura nos duela, tenemos derecho a manifestarlo, no ver nuestra $\mathrm{vOZ}$ callada ni avergonzarnos: \#OrgulloLoco

Así pues, los conceptos "prejuicio social" y "diagnóstico" como dominio destino de metáforas están relacionados por el hecho de tener un dominio de origen común, "una marca", y puede inferirse que ambos tipos de señal ignominiosa están conectados (para el activista) por una relación de causa-efecto: el etiquetado del diagnóstico causa la estigmatización, es decir, prejuicio social.

De esta manera, la metáfora "el prejuicio social es una marca" refiere a la relación directa que existe entre diagnóstico y estigma. 
Al decir de Goffman, el estigma se convierte en un atributo profundamente desacreditador $^{(26)}$ que totaliza al sujeto de modo negativo. El dolor social amplio que se genera en la locura tiene relación directa con dicho estigma. Este produce ocultación, rechazo y aislamiento, pero pocas veces se reconoce este sufrimiento social:

La noción de estigma es clave en este sentido, la estigmatización del afectado se materializa en un rechazo a priori, en una desautorización sistemática, en todos los aspectos, de su cotidiano, que se transforma en uno de los ejes productores de mayor sufrimiento. $Y$ el estigma es un producto esencialmente social. Surge de esa dificultad comunal de lidiar con la diferencia, que en ocasiones actúa como agudizante y cronificador de la problemática. Por estas razones, toda aproximación al sufrimiento mental debería partir de la comprensión del fenómeno en sus dimensiones sociales, culturales, biológicas, psíquicas, subjetivas, etc. ${ }^{(27)}$

Además, el primer ingreso y su consiguiente primer diagnóstico tiene para algunos autores ${ }^{(28,29,30,31)}$ las características de un rito de paso: "Crea una discontinuidad con la vida anterior y por ese medio anuncia la presentación de una nueva etapa evolutiva"(31). En síntesis, hay un antes y un después del diagnóstico que produce una fractura biográfica. Una especie de escalada de consecuencias nefastas para los sujetos. Para Elizur y Minuchin $^{(31)}$, lo que llamamos enfermedad mental es un proceso complejo que se explica por la participación de individuos, familias y comunidades en una serie de intercambios destructivos. Por ello, el fenómeno de la psiquiatrización es incomprensible sin considerar estos factores sociales y culturales que explican el plus de sufrimiento asociado a la reacción comunitaria que conlleva la etiqueta diagnóstica.

Este impacto es global e ininterrumpido. Se trata de lo que Correa-Urquiza et al. ${ }^{(32)}$ han denominado paciente total: la reducción del sujeto diagnosticado a la noción de enfermo absoluto. La etiqueta captura la totalidad de la existencia hasta el punto que supone un punto de inflexión en sus biografías. Aquí, la categoría diagnóstica coloniza y asfixia la totalidad subjetiva de la persona, la "marca" captura, clausura la complejidad identitaria. Y de lo que se trata, al decir de las metáforas utilizadas, es de des-amarrarse del estigma, interpelar el prejuicio. Y para ello, es necesario cuestionar el uso de las categorías biomédicas como entidades totalizantes que operan sobre las vidas y subjetividades de los individuos. Hoy, el estigma está en el diagnóstico y en las capturas que este produce sobre los sujetos. De ahí, la necesidad de recuperar la polisemia del concepto locura como manera y camino de instituir una cierta capacidad de agencia.

\section{"Metáforas de la comunicación"}

Como siguiente dominio de destino en volumen cuantitativo, los aspectos comunicativos se revelan también importantes para el activismo. La comunicación (o su reverso, la incomunicación) con la sociedad se conceptualizan fundamentalmente en términos de visibilidad/ocultación (9a) o de proximidad/ distancia (9b), siendo los prejuicios una barrera que impide el acercamiento (9c).

(9)

a. Cada quién que viva y sienta como quiera, no era eso la diversidad, respetar la diferencia? Pues quien sienta orgullo genial. Es más constructivo que la vergüenza que hemos sentido, por haber sido invisibles. Gracias por luchar @tomcorom A tods fuerza para el \#OrgulloLoco

b. El movimiento en 1a persona ha despertado, ios acercáis a nosotras?: @ FlipasGAM, @Col_InsPIRADAS, @orguIlo_loco, @OrgulloLocoMad Por favor, no más \#DiaMundialDeLaSaludMental sin nosotras, \#nadaparanosotrassinnosotras \#MeMad \#saludmentalpositiva \#sinfiltros \#notehagaselloco 
c. Ven con nosotros a celebrar el día del Orgullo Loco. Aprendamos a romper barreras. \#20MYoVoy

Otras conceptualizaciones habituales, aunque no tan abundantes como las anteriores, pero que son interesantes para caracterizar el lenguaje metafórico del Orgullo Loco se centran en la concepción de espacios cerrados tanto del trastorno (10a) como de la superestructura social denominada sistema (10b).

a. Si estás recién llegada a la locura y los psiquiatras te dicen q no hay otra salida, te agarras a un clavo ardiendo. Luego $t$ quemas, claro q t quemas, pero ¿había otra opción?

b. Si no estás de acuerdo con el sistema lo mejor es salir y poner en marcha tu proyecto fuera.

Como hemos visto, a grandes rasgos destaca en este corpus la poca incidencia de metáforas del trastorno como entidad per se, frente a la gran abundancia de metáforas relativas a aspectos vitales, sociales y comunicativos.

En primer lugar, la metáfora dominante "comunicar/incomunicar es mostrarse/esconderse" refiere al binomio movimiento/reclusión asociado históricamente a la locura. Según Martínez-Hernáez,

...en la historia de Europa la locura ha venido unida a dos condiciones o atributos: el tránsito y el confinamiento. El primero adquiere una de sus mejores representaciones en El Quijote, en donde el insensato se ve abocado a un errar continuo [...]. El segundo incluye entre otras fórmulas la imagen del manicomio como lugar de reclusión no se sabe a ciencia cierta de qué - ¿la sinrazón?, la falta de sentido común, el caos, el desorden, el miedo, la disidencia, la diferencia?-, pero que persiste durante siglos como una forma de control social. ${ }^{(33)}$
El autor afirma que ambos atributos -movimiento y reclusión- se combinan de manera frecuente y persistente formando parte del imaginario social de la locura. El fenómeno conocido como "La nave de los locos" o stultifera navis da cuenta de ello. El peso de este imaginario social alimenta la confusión entre los modos sociales de tratar la locura y la locura en sí misma. De este modo, el gran período de encierro queda incrustado en la mirada social contemporánea. La ocultación de la locura forma parte de sus mismos orígenes (el loco es el que debe ser encerrado) y, en consecuencia, su desocultación se concibe como estrategia de resistencia. La exclusión y coto al tránsito, primero, y el encierro, después, formarán parte del palimpsesto de la locura. Por ello, para Martínez, la stultifera navis persiste más tarde en el asilo, del mismo modo que, para Foucault ${ }^{(34)}$, la exclusión y el orden quedarán unidos al gran encierro:

\section{El manicomio instaura un sistema de reclusión sedentario, pero que al igual que los barcos de los locos supone la concentración de los insensatos en un espacio físico que es purificador y a la vez incierto. ${ }^{(33)}$}

En la cita debe entenderse "purificador" como terapéutico, y esta es la razón que termina produciendo una adhesión (confusión) entre el encierro y la locura: el loco es el que no puede vagar libremente y debe ser encerrado. Los modos de gestión contemporánea de la locura no borran estas significaciones previas, sino que vienen a sumarse en configuraciones semánticas más amplias. Por lo tanto, comunicar no es solo nombrar sino sacar al exterior, visibilizar algo que estaba oculto.

El problema social no es tanto la locura en sí misma, sino la errancia que entraña en sí misma. Contenerla e inmovilizarla formará parte de su propia historia. La locura es entonces desmesura e incontinencia, pero ¿desmesura de qué? La respuesta la encontramos en otro de los dominios de destino analizados: la "comunicación/incomunicación" del dolor y el sufrimiento. La desmesura, por tanto, refiere a este sufrimiento. Sabemos que 
en nuestras sociedades occidentales existen límites para la demostración del dolor ${ }^{(35)}$. ¿Hasta dónde está permitido sufrir? Estos límites son los que marcan lo que es nombrable y lo que no. Modificar o cuestionar estos límites y ampliar sus horizontes es otra de las batallas del activismo contemporáneo. Así, la dialéctica entre movimiento y contención condensa también la relación entre libertad y reclusión. Libertad de movimiento, pero también libertad de nombramiento. Transitar refiere, entonces, a deambular, experimentar y sentir. Ocultar lo sentido y experimentado es algo recurrente para las personas diagnosticadas al ser conocedoras de la capacidad coercitiva del sistema. La imposibilidad de comunicar libremente las propias experiencias inusuales (alucinaciones, delirios...) revierte en un mayor sufrimiento y aislamiento social. Comunicar desde la locura refiere entonces a una imposibilidad asentada por la propia lógica manicomial hegemónica:

\begin{abstract}
La presunción de que la locura ya viene unida de forma irresoluble a la confusión y al error genera una relación de poder en la que diga lo que diga o haga lo que haga el afectado su comportamiento siempre estará en contra suya. Tanto la afirmación como la negación de su locura siempre responderá a las expectativas del terapeuta. $Y$ es que una vez incluido en el catálogo de los locos el círculo se ha cerrado ante todo tipo de argumentos. ${ }^{(33)}$
\end{abstract}

La locura interpela y deja en evidencia el constructo colectivo de normalidad que se instaura a modo de ley, pone en evidencia las arbitrariedades de lo dado, del sentido común. Y es por eso quizás, que se ha vuelto socialmente necesario encuadrarla, encerrarla para ajustarla a lo definido como "normal". Silenciarla. La posibilidad de comunicar, de decir, es aquí y entonces una manera de producir resistencias, de oponerse al silencio y al encuadre unidireccional que asfixia vidas e itinerarios. La comunicación es libertad.

Al mismo tiempo, la idea de comunicación pone de manifiesto dos situaciones distintas pero que se complementan. Por un lado, la evidencia de la falta de comunicación real entre profesionales y pacientes; la percepción de que hay solo un monólogo biomédico sobre el sufrimiento psíquico, decíamos y, por otro, la necesidad de establecer nuevas condiciones para el diálogo, condiciones de respeto, escucha, legitimidad y acción conjunta. Lo cual significa, a su vez, habilitar otros contextos en los que se trabaje desde una des-fosilización/des-cosificación mutua por parte de los sujetos del diálogo.

\section{CONCLUSIONES}

A nuestro entender, el Día del Orgullo Loco puede leerse en términos de espacios e itinerarios para la re-semantización de lo dado, como territorio de resistencia, de conquista de derechos y producción de nuevas maneras de pensar y problematizar el sufrimiento psíquico. La celebración es también un campo de resonancias intersubjetivas, es un espacio de producción de lo transubjetivo, en términos de Alicia Stolkiner ${ }^{(36)}$. Se transforma en una plaza íntima en movimiento ${ }^{(37)}$ un umbral, un tercer lugar ${ }^{(38)}$, un territorio para la legitimación de lo colectivo que produce la posibilidad de dar lugar a nuevas significaciones ya desligadas del cuerpo de lo biomédico. De la misma manera el movimiento vinculado a la Diversidad Funcional ha abierto nuevos campos de resignificación con relación al concepto de "Discapacidad". También el movimiento Queer en la década de 1970 y el Crip en la de 1990 recuperaba y recolocaba aquello que inicialmente se planteaba como ofensivo. La idea de locura rescata aquí una apertura semántica que oxigena la clausura que produce la noción de enfermedad mental. La idea de locura des-fosiliza lo que el concepto de enfermedad cierra y el Día del Orgullo Loco se evidencia como una oportunidad para la liberación de una cierta captura semántica de cuerpos y destinos vitales. No puede existir liberación semántica sin otro ordenamiento y otras producciones lingüísticas. El campo del lenguaje es territorio de 
generación de sentidos y es por este motivo que en muchos activismos de la diferencia los nombramientos son un elemento clave para la recuperación de la agencia política.

Si partimos de la idea de que en el ámbito de la salud mental las categorías médicas reducen, por un lado, la totalidad compleja de la experiencia del sufrimiento a una descripción sintomatológica y, por otro, colonizan la identidad y la subjetividad de las personas, el Día del Orgullo Loco se evidencia como una oportunidad para la generación de un corpus disidente, una communitas circunstancial, de performatividad momentánea que promueve el desacato y la recuperación de una cierta legitimidad para nombrarse, pensarse. Lo que está realmente en juego en dichos nombramientos es el sujeto de la enunciación, es decir, la posibilidad de generar nuevas narrativas sobre lo vivido en primera persona. Ello conlleva a que las palabras dejen de recaer sobre cuerpos-depósito para pasar a ser cuerpos-texto que son reescritos. Las relaciones de poder entre nombradores y nombrados se mueven en el terreno lingüístico del "haber sido excesivamente hablados por otros". Revertir esta situación es uno de los propósitos de la conquista del lenguaje y en ello resulta central la subversión de las palabras, la inversión de su sentido, la (re)apropiación del insulto o el desvelo del palimpsesto de la propia etimología.

Al mismo tiempo, es necesario pensar el Día del Orgullo Loco en tanto contexto/territorio que habilita y posibilita en su accionar múltiple y multitudinario, la emergencia de aquellos otros discursos que evidencian la disidencia. El Día del Orgullo Loco es, a la vez, una suerte de "límite habitable"(37), de "tercer espacio" en términos de Homi Bhabha ${ }^{(38)}$, de umbral; un momento/instancia/lugar liminar, flexible, abierto desde donde es posible el cuestionamiento y la recuperación de un lugar históricamente negado.

Si seguimos a Michel Agier ${ }^{(39)}$ podemos pensar que "el sujeto surge entonces en situación" y existe "al tomar distancia de su condición social, de la identidad que le es asignada (étnica, racial, humanitaria) y eventualmente de un sí mismo que sufre". Para el autor "donde hay subjetivaciones otras, hay aparición de un sujeto otro". El Día del OrguIlo Loco es definitivamente un contexto para la re-subjetivación, es la posibilidad de ser un sujeto otro, que existe, que toma distancia de las lógicas biomédicas que lo nombran y se constituye desde nuevos lugares. Si seguimos a Delgado podemos decir que:

Es en los territorios sin amo, sin marcas, sin tierra, donde se da la mayor intensidad de informaciones, donde se interrumpen e incluso se llegan a invertir los procesos de igualación entrópica y donde se producen lo que Rubert de Ventós Ilamaba "curiosos fenómenos de frontera" en los que el contacto entre sistemas era capaz de suscitar la formación de verdaderos islotes de vida y de belleza". Honoré de Balzac había dicho lo mismo de otro modo: "sólo hay vida en los márgenes". Convicción última de que lo más intenso y más creativo de la vida social, de la vida afectiva y de la vida intelectual de los seres humanos se produce siempre en sus límites. ${ }^{(40)}$

Las metáforas analizadas funcionan en la traducción de un estado intransmisible, ayuda en este ámbito a recuperar las posibilidades de una subjetividad negada, abrirse a la enunciación del dolor. Las metáforas dan cuerpo a lo que, de otro modo, no puede ser nombrado, es el lugar para la traducción de lo complejo de la experiencia de la aflicción y para el grito que disiente y busca alternativas. Las metáforas surgen aquí en forma de rugido, de búsqueda de la emancipación que no renuncia a la necesidad del cuidado. Las metáforas aquí no son nunca en tanto producto del trastorno mental, sino una manera de neutralizar colectiva e individualmente lo que la sociedad produce y ejecuta en términos de hegemonías sobre las Ilamadas enfermedades mentales. Es la materialización de una lucha por la hegemonía del relato.

A través de las metáforas se observa cómo es vivida y expresada la celebración del Día del Orgullo Loco: como instancia para desenfermar identidades; como una oportunidad 
para quebrar la captura de la vida por parte de las nosologías psiquiátricas, promover la desmedicalización de los procesos de la vida, oxigenar el mundo vital abriéndose a la pluralidad semántica de la locura y, sobre todo, abriendo los diques que contienen la experiencia de la aflicción entre los muros conceptuales de la enfermedad, prácticas emancipadoras para una subjetividad acorralada.

Como investigadores, cabe decir que el análisis de las metáforas da cuerpo y contundencia a la reflexión posible sobre el mo- mento que hoy atraviesa el movimiento "en primera persona" y la necesidad de generar nuevos contextos de diálogo y complicidad entre saberes expertos y saberes profanos. El Día del Orgullo Loco es un espacio transitorio en su andadura, pero permanente en términos de que va soltando la simiente de las contestaciones posibles. $Y$ es aquí y ahora que la idea de locura vuelve a surgir como un concepto que actúa y se articula como pivote, eje para la producción de esas nuevas significaciones quizás emancipadoras.

\section{AGRADECIMIENTOS}

Esta investigación se ha llevado a cabo en el marco del proyecto MOMENT: Metáforas del trastorno mental grave. Análisis del discurso de personas afectadas y profesionales de la salud mental (Referencia: FFI201786969-R), financiado por la Agencia Estatal de Investigación (AEI) de España y el Fondo Europeo de Desarrollo Regional (FEDER), en la Convocatoria 2017 "Retos Investigación", del Programa de I+D+i Orientada a los Retos de la Sociedad.

\section{REFERENCIAS BIBLIOGRÁFICAS}

1. Huertas R. Letras locas: Del yo disidente al activismo en primera persona. e-atopos, 2019;5:3-19.

2. Castel R. El tratamiento moral: Terapéutica mental y control social en el siglo XIX. En: García R, (ed.). Psiquiatría, antipsiquiatría y orden manicomial. Barcelona: Barral; 1975. p. 71-96.

3. Foucault M. El poder psiquiátrico. Madrid: Akal; 2005.

4. Correa-Urquiza M. La condición del diálogo: Saberes profanos y nuevos contextos del decir. Revista de la Asociación Española de Neuropsiquiatría. 2018;38(134):567585. doi: 10.4321/s0211-57352018000200012.

5. Coll-Florit M, Climent S, Correa-Urquiza M, Hernández E, Oliver A, Pié A. MOMENT: Metáforas del trastorno mental grave: Análisis del discurso de personas afectadas y profesionales de la salud mental. Procesamiento del Lenguaje Natural. 2018;61:139-142. doi: 10.26342/2018-61-17.

6. Lakoff G, Johnson M. Metaphors we live by. Chicago: University of Chicago Press; 1980.

7. Climent S, Coll-Florit M. La metáfora conceptual en el discurso psiquiátrico sobre la esquizofrenia. Ibérica: Revista de la Asociación Europea de Lenguas para Fines Específicos (AELFE). 2017;34:187-208.
8. Coll-Florit M, Climent S. A new methodology for conceptual metaphor detection and formulation in corpora: A case study on a mental health corpus. SKY Journal of Linguistics. 2019;32:43-74.

9. McMullen LM, Conway JB. Conventional metaphors for depression. En: Fussell SR, (ed.). The verbal communication of emotions. Mahwah: Erlbaum; 2002. p. $167-$ 118.

10. Charteris-BlackJ. Shattering the Bell Jar:Metaphor, gender, and depression. Metaphor and Symbol. 2012;27(3): 199-216. doi: 10.1080/10926488.2012.665796.

11. Semino E. Metaphor in discourse. Cambridge: Cambridge University Press; 2008

12. Coll-Florit M, Miranda X, Climent S. Metáforas de la esquizofrenia: Un estudio sobre el discurso de afectados y profesionales. RESLA, Revista Española de Lingüística Aplicada. 2019;32(1):1-31. doi: 10.1075/resla.16003.col.

13. Berry N, Lobban F, Belousov M, Emsley R, Nenadic G, Bucci S. \#WhyWeTweetMH: Understanding why people use Twitter to discuss mental health problems. Journal of Medical Internet Research. 2017;19(4):e107. doi: 10.2196/jmir.6173.

14. Lachmar E, Wittenborn A, Bogen KW, McCauley HL. \#MyDepressionLooksLike: Examining public discourse about depression on Twitter. JMIR Mental Health, 2017;4(4):e43.

15. Alcaraz E, Martínez MA. Diccionario de lingüística moderna. Barcelona: Ariel; 1997.

16. Littlemore J, Tagg C. Metonymy and text messaging: A framework for understanding creative uses of metonymy. Applied Linguistics. 2018;39(4):481-507. doi: 10.1093/applin/amw018.

17. Pié A, Correa-Urquiza M, Martínez-Hernáez A. Salud mental colectiva: miradas, encuentros, diálogos. Barcelona: UOC. En prensa: 2020. 
18. Gramsci A. Lettere del carcere 1926-1937. Palermo: Sellerio; 1996.

19. Martínez-Hernáez A. Vidas apagadas: el sentido común de la locura y la locura del sentido común. Conferencia pronunciada en: I Seminario Internacional sobre Salud Mental e Intervención Comunitaria: Revisando la idea de locura; 15 de septiembre de 2012; Lanzarote, España.

20. Cano O. Desde adentro: ensayo autoetnográfico de una paciente internada en el Hospital Psiquiátrico de Asunción, Paraguay. En: Cea JC, (ed.). Por el derecho a la locura: La reinvención de la salud mental en América Latina. Santiago: Proyección; 2018. p. 115-126.

21. Cea JC, (ed.). Por el derecho a la locura: La reinvención de la salud mental en América Latina. Santiago: Proyección; 2018.

22. Fredes R. El derecho a la locura en Chile: Construyendo desde los márgenes y en las grietas del sistema. En: Cea JC, (ed.). Por el derecho a la locura: La reinvención de la salud mental en América Latina. Santiago: Proyección; 2018. p. 91-110.

23. Freire P. Pedagogía del oprimido. Montevideo: Tierra Nueva. México: Siglo XXI Editores; 1985.

24. Martínez-Hernáez A. El síntoma y sus interpretaciones: en los límites de la Psiquiatría y la Antropología de la medicina contemporáneas [Tesis doctoral]. Barcelona: Universitat de Barcelona; 1994.

25. Menéndez EL. El modelo médico hegemónico: transacciones y alternativas hacia una fundamentación teórica del modelo de autoatención en salud. Arxiu d'Etnografia de Catalunya. 1984;3:83-119.

26. Goffman E. El estigma. La identidad deteriorada. Buenos Aires: Amorrortu; 1989.

27. Correa-Urquiza M, Pié A. Capturas e itinerarios de la locura. Barcelona: UOC; 2017.

28. Scheff T. El rol de enfermo mental. Buenos Aires: Amorrortu; 1966.
29. Parsons T. The social system. London: Taylor and Francis e-Library; 1951.

30. Goffman E. Internados: Ensayos sobre la situación social de los enfermos mentales. Buenos Aires: Amorrortu; 2001.

31. Elizur J, Minuchin S. La locura y las instituciones: familias, terapia y sociedad. Barcelona: Gedisa; 2006.

32. Correa-Urquiza M, Silva TJ, Belloc MM, MartínezHernáez A. La evidencia social del sufrimiento: Salut mental, políticas globales y narrativas locales. Quaderns de I'Institut Català d 'Antropologia. 2006;22(Número especial):47-69.

33. Martínez A. ¿Has visto cómo llora un cerezo? Pasos hacia una antropología de la esquizofrenia. Barcelona: Universitat de Barcelona; 1998.

34. Foucault M. Historia de la locura en la época clásica, 1 y 2. Madrid: Fondo de Cultura Económica; 2002.

35. Pié A. La insurrección de la vulnerabilidad: Para una pedagogía de los cuidados y la resistencia. Barcelona: Universitat de Barcelona; 2019.

36. Stolkiner A. La interdisciplina: entre la epistemología y las prácticas. Revista El Campo Psi. 1999;3:10.

37. Correa-Urquiza M. Radio Nikosia. La rebelión de los saberes profanos: Otras prácticas, otros horizontes para la locura. Madrid: Grupo 5; 2015.

38. Bhabha H. El lugar de la cultura. Buenos Aires: Manantial; 1998.

39. Agier M. Pensar el sujeto, descentrar la antropología. Cuadernos de Antropología Social. 2012;(35):9-27. doi: 10.34096/cas.i35.1360

40. Delgado M. Sociedades movedizas: Pasos hacia una antropología de las calles. Barcelona: Anagrama; 2007.

FORMA DE CITAR

Correa-Urquiza Vidal M, Pié Balaguer A, Coll-Florit M, Hernández i Encuentra E, Climent Roca S. Orgullo loco y metáforas para una disidencia: un análisis lingüístico y simbólico. Salud Colectiva. 2020;16:e2886. doi: 10.18294/ sc. 2020.2886 .

Recibido: 15 jun 2020 | Aprobado: 17 jul 2020 | Publicado en línea: 7 ago 2020

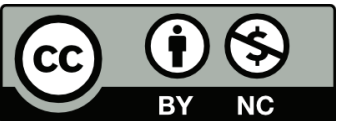

Esta obra está bajo una licencia de Creative Commons Reconocimiento-NoComercial 4.0 Internacional. Reconocimiento - Permite copiar, distribuir y comunicar públicamente la obra. A cambio, se debe reconocer y citar al autor original. No Comercial - Esta obra no puede ser utilizada con finalidades comerciales, a menos que se obtenga el permiso. 